\title{
NOUVELLE
}

\section{Nouveaux déterminants génétiques des traits glycémiques}

\section{L'apport de l'association génomique}

Nabila Bouatia-Naji ${ }^{1,2,3}$

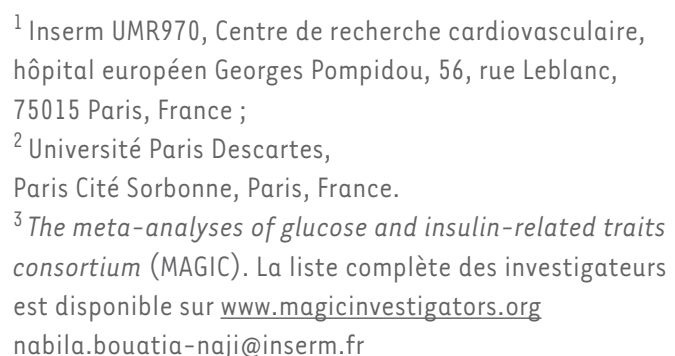
nabila.bouatia-naji@inserm.fr

> Au cours des cinq dernières années, la génétique des maladies multifactorielles a connu une phase de foisonnement sans précédent, portée essentiellement par la publication d'un nombre impressionnant d'études d'association génomique (GWAS : genome-wide association studies) (voir Glossaire), avec à ce jour plus de 1717 articles portant sur la génétique de divers traits et maladies humaines (www.genome.gov/gwastudies, septembre 2013).

MAGIC, pour The meta-analyses of glucose and insulin-related traits consortium, est un groupe de recherche collaboratif international constitué par des épidémio-généticiens, des médecins diabétologues et endocrinologues et des généticiens statisticiens ${ }^{1}$. Les chercheurs MAGIC mutualisent ainsi les données GWAS individuelles obtenues dans de grandes populations de patients non diabétiques, exemptes de toute les complications métaboliques et cardiovasculaires qui caractérisent les patients diabétiques. Cette approche s'est montrée très efficace pour mettre en évidence des gènes qui contrôlent l'homéostasie glucidique de façon indépendante du risque du diabète de type 2 (DT2). Dans cette nouvelle, j'aborderai les résultats majeurs de la dernière publication MAGIC [1] qui apporte de nouveaux éclairages sur les voies biologiques impliquées dans le déterminisme génétique de la glycémie et de l'insulinémie.

\footnotetext{
${ }^{1}$ www.magicinvestigators.org
}

\section{Objectif et stratégie de l'étude}

MAGIC a été à l'origine de plusieurs métaanalyses qui ont identifié jusqu'à présent 16 locus associés avec la glycémie à jeun, deux avec l'insulinémie et cinq avec la glycémie postprandiale [2, 3]. Cependant, si la composante génétique de ces traits - ou héritabilité - est estimée entre 30 et $60 \%$, ces locus ne peuvent suffire à expliquer la part génétique de ces traits. C'est la raison pour laquelle la metabochip a été développée. II s'agit d'une puce de génotypage à façon contenant environ 200000 polymorphismes de type SNP (voir Glossaire). Le contenu de la metabochip a été défini par un comité de chercheurs qui s'intéressent à la génétique de maladies multifactorielles liées au risque cardiovasculaire. Le but de cet outil est la réalisation d'études d'association génétique de très grande envergure à moindre coût. MAGIC a contribué à la metabochip à hauteur d'environ 6000 variants $[2,3]$. Cette étude a été réalisée chez un total de 133010,108557 et 42854 individus respectivement pour la glycémie à jeun, l'insulinémie à jeun et la glycémie postprandiale, tous d'ascendance européenne [1].

\section{Un nombre élevé de locus contrôlent} la génétique des traits glycémiques Nous avons identifié 41 nouveaux locus qui modulent les traits glycémiques portant le nombre total à 53 locus confirmés au seuil génomique (voir Glossaire). À ce stade, 36 locus indépendants expliquent ensemble 4,8\% de la variance de la glycémie ; 19 locus expliquent $1,2 \%$ de celle de l'insulinémie et neuf locus expliquent $1,7 \%$ de celle de la glycémie postprandiale. Si malgré les effectifs utilisés, ces proportions de variances expliquées semblent faibles, ceci est dû au caractère hautement polygénique des traits glycémiques, et d'autres locus ayant des effets au moins similaires à ceux identifiés jusqu'à présent restent à découvrir en adoptant d'autres stratégies.

Les déterminants de la glycémie et de l'insulinémie augmentent en majorité le risque de DT2

L'une des motivations majeures de l'étude de la génétique des traits glycémiques est de savoir si ces locus augmentent le risque de DT2, de l'obésité, et le risque cardiovasculaire. Ces analyses permettent de dresser un (des) profil(s) d'association de ces nouveaux locus, ce qui permettrait, à long terme, de classer prospectivement les futurs patients diabétiques et leurs complications.

Parmi les 36 locus associés à une augmentation de la glycémie à jeun, 22 (> 60 \%) le sont aussi avec un risque plus élevé de DT2 ; huit locus sur neuf pour la glycémie postprandiale; les allèles associés avec l'augmentation de la glycémie étant systématiquement à risque pour le DT2 [1] (Figure 1). Il est important de noter que nous n'avons observé aucune corrélation entre l'amplitude de l'effet sur la variabilité de la glycémie et l'association avec le DT2. Les deux exemples les plus frappants étant ceux des locus G6PC2 (glucose-6-phosphatase, catalytic, 2) et MTNRIB (melatonin receptor $1 B$ ), dont 


\section{GLOSSAIRE}

Association génomique : ce terme désigne les études d'association en épidémio-génétique qui s'intéressent à l'analyse de l'ensemble des variants génétiques connus. Les données de génotypage sont générées par des puces de génotypages commerciales dont le contenu est variable (100000 variants pour les premières générations et jusqu'à 4 millions de variants pour les puces les plus récentes). L'étude consiste à tester si chacun de ces variants génétiques est associé à une maladie de la variabilité interindividuelle d'un trait quantitatif, ici la glycémie et l'insulinémie.

SNP (single nucleotidic polymorphism) : II s'agit d'un terme qui désigne les polymorphismes génétiques ponctuels et les très petites délétions/insertions. Les SNP peuvent être fréquents ou rares dans les populations humaines et leur fréquence est dépendante de l'origine ethnique. Dans le cadre des associations génomiques, ce sont notamment les SNP fréquents qui sont étudiés (fréquence supérieure à $5 \%$ dans la population générale). Seuil génomique d'association: il s'agit d'un niveau de significativité établi à une p-value $<5 \times 10^{-8}$ pour déclarer l'association d'un locus comme significative au niveau génomique. Quand un grand nombre de tests statistiques sont réalisés dans un même échantillon, les chances que ces tests soient significatifs par hasard au seuil conventionnel de 0,05 est élevé et il est proportionnel au nombre de tests réalisés. Dans une association génomique, on estime que le génome humain compte environ l million de polymorphismes indépendants. De ce fait, une étude GWAS consiste en 1 million de tests différents et la $p$-value corrigée comme stipulé par Bonferroni pour des tests multiples est donc $0,05 \div 10^{6}=5 \times 10^{-8}$.

les effets sont presque identiques sur la glycémie $(0,07 \mathrm{mmol} / \mathrm{l}$ par allèle) mais sans effet sur le risque de DT2 pour G6PC2 $[2,5,6]$. En ce qui concerne l'insulinémie, pour laquelle deux locus étaient connus jusqu'à présent, la situation est comparable avec 13 locus sur 19 associés avec un risque plus élevé de DT2. Globalement, l'ajustement pour la corpulence, fortement corrélée avec l'insulinémie à jeun, a diminué le signal de l'association pour la plupart des locus, voire a fait disparaître le signal dont l'effet sur la résistance à l'insuline est totalement attribué à l'effet sur la corpulence (ex. locus FTO, fat mass and obesity associated). En parallèle, l'ajustement par I'IMC (indice de masse corporelle) a contribué à diminuer la variance de l'insulinémie, ce qui est favorable à une meilleure détection d'un effet génétique. Ainsi, cinq locus associés au niveau génomique (voir Glossaire) avec l'insulinémie l'ont été seulement après ajustement pour la corpulence. En effet, ces locus particuliers sont légèrement associés $(p<0,05)$ avec un IMC plus bas, ce qui pourrait expliquer que leur association avec l'insulinémie soit démasquée seulement une fois l'effet sur l'obésité neutralisé ; ce résultat concerne les locus PPARG (peroxisome proliferator-activated receptor), ANKRD55 (ankyrin repeat domain 55)/MAP3K1, PDGFC (plateletderived growth factor C), FAMI3A (family with sequence similarity 13 , member $A$ ) et UHRFIBPI (ubiquitin-like containing PHD And RING finger domains 1-binding protein). D'une façon générale, nous avons observé une très forte concordance de l'association des locus de l'insulinémie avec l'obésité et les traits lipidiques. En effet, 13 des 19 locus (soit $68 \%$ ) qui augmentent l'insulinémie sont corrélés à des niveaux plus bas du HDL (high density lipoproteins)- cholestérol, neuf locus le sont avec des niveaux plus élevés de triglycérides, et quatre sont corrélés à un rapport tour de taille/tour de hanches plus élevé, un indicateur anthropométrique d'une obésité centrale qui prédit fortement le risque cardiovasculaire.

\section{Enrichissement en gènes candidats autour des loci associés}

Comme dans toute exploration par GWAS, les associations décrites concernent majoritairement des variants génétiques intergéniques et la façon dont ces variants agissent pour moduler ces traits demeure inconnue. Pour aiguiller notre compréhension d'un mécanisme moléculaire et/ ou physiologique, nous avons utilisé la méthode GRAIL (gene relationship across implicated loci) [7], qui utilise les données de la littérature pour trouver un lien entre les gènes autour des locus associés en GWAS, et la méthode MAGENTA : meta-analysis gene-set enrichment of variant associations, pour tester l'enrichissement en association de gènes/voies biologiques candidats [8]. Ainsi, nous avons établi que les locus associés avec les traits glycémiques sont situés à proximité de gènes dont le lien avec l'homéostasie glucidique est plausible, et ce de façon non aléatoire. Par exemple, pour la glycémie à jeun - le trait pour lequel nous avons le plus grand nombre de locus $(N=36)$-, les analyses GRAIL ont montré une forte connectivité parmi les gènes des locus associés avec les mots clés «glucose», «insulin », «pancreatic » et «diabetes » [1]. Certains gènes codent pour des facteurs de transcription impliqués dans le développement pancréatique (ex. PDXI [pancreatic and duodenal homeobox 1], FOXA2 [forkhead box A2], et PROX1 [prospero-related homeobox gene]), alors que d'autres sont impliqués dans la fonction bêta-pancréatique de sécrétion de l'insuline (SLC2A2 [solute carrier family 2 (facilitated glucose transporter), member 2], GCK [glucokinase], PCSK1 [proprotein convertase subtilisin/kexin type 1]). D'autre part, tous les gènes à proximité des locus associés aux traits glycémiques ne peuvent être liés à ce stade avec la biologie de I'homéostasie glucidique. C'est le cas du locus DNLZ/CARD9 (caspase recruitment domain family, member 9), connu pour augmenter le risque de la maladie de Crohn et de la rectocolite hémorragique pour lesquelles aucun gène candidat n'a été identifié ; c'est aussi le cas du locus où se situe GLS2 (phosphateactivated mitochondrial glutaminase) 


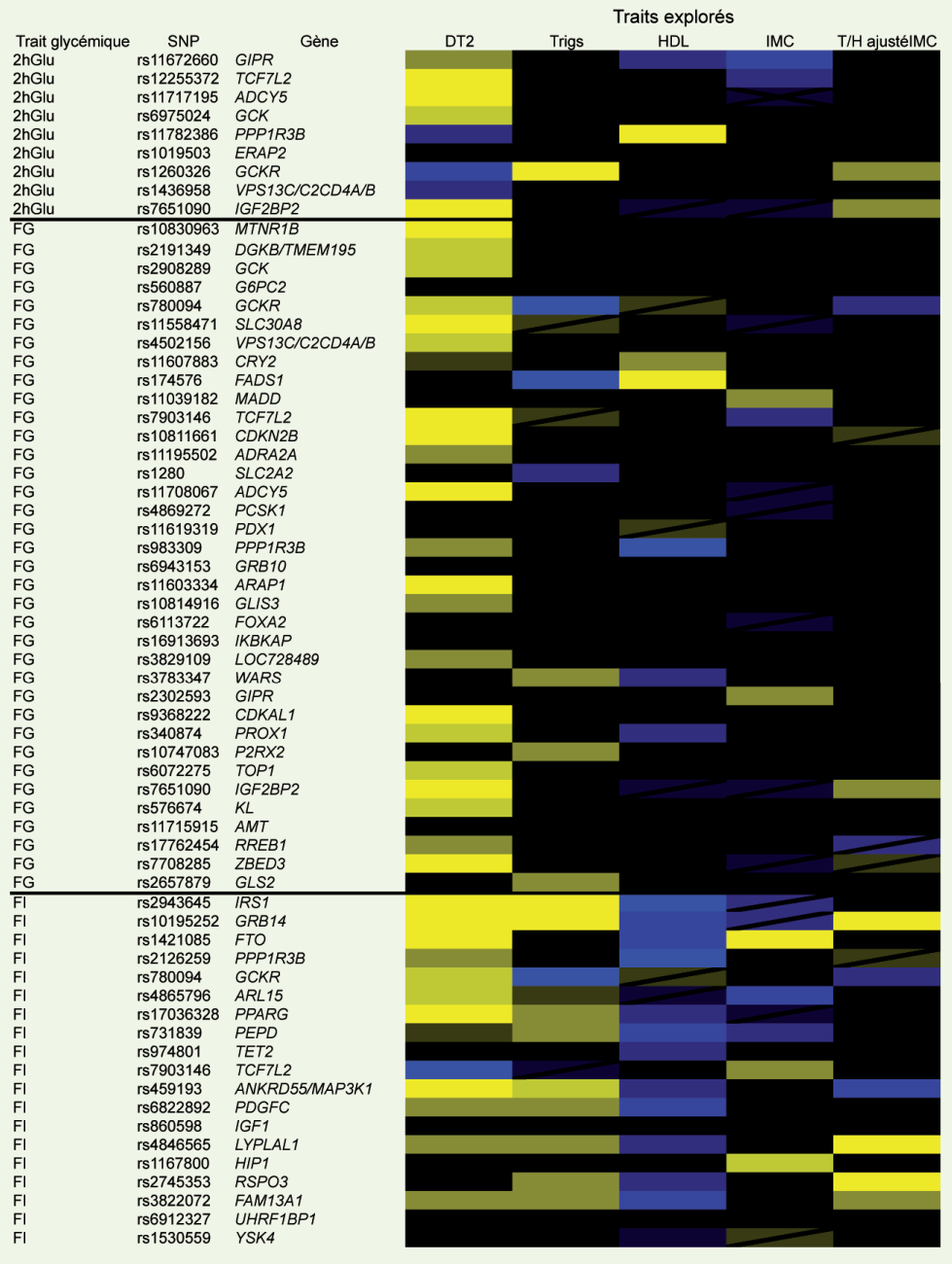

qui code pour une enzyme impliquée dans le métabolisme du glutamate, ou encore de SPRYD4 (SPRY domain containing 4), de fonction inconnue [1]. Ces loci constituent donc de nouvelles pistes qui méritent une exploration biologique plus complète afin d'identifier le lien des gènes à proximité avec la régulation de la glycémie et de l’insulinémie.

\section{Conclusion}

Cette étude du groupe collaboratif MAGIC, réalisée chez plus de 133000 individus, a permis la confirmation et/ou l'identification de 53 déterminants génétiques de la glycémie et de l'insulinémie, dont 33 modulent également le risque de DT2. Autour de ces locus, de nombreux gènes candidats ont été révélés, notamment certains gènes impliqués dans le développement pancréatique (par exemple PDX1, FOXA2 et $P R O X 1$ ), dans le maintien de la fonction sécrétrice de la cellule bêta-pancréatique (SLC2A2, GCK et $P C S K 1$ ) et dans la signalisation de I'insuline (par exemple IRS1, GRB14). Le futur de l'investigation sur la génétique des traits glycémiques sera consacré à la recherche des variants causatifs au sein des locus confirmés, d'une part par une approche de cartographie fine multiethnique à plus grande échelle, et, d'autre part, par l'étude du rôle de variants génétiques plus rares, dont l'apport à l'architecture génétique de ces traits reste à déterminer. $\diamond$

New genetic determinants of glycemic traits: insights in biological pathways of glucose homeostasis

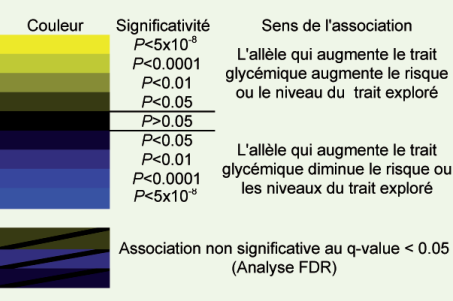

Figure 1. Représentation de l'association entre les locus associés avec les traits glycémiques et le risque de DT2, les niveaux de HDL-cholestérol, de triglycérides, l'indice de masse corporelle (IMC) et le rapport tour de taille/tour de hanches (T/H) ajusté par l'IMC. Les locus associés avec les traits explorés sont surlignés en jaune quand le locus qui augmente le trait glycémique augmente aussi le trait exploré, et en bleu s'il s'agit d'un effet opposé.

\section{LIENS D'INTÉRÊT}

Les auteurs déclarent n'avoir aucun lien d'intérêt avec les données publiées dans cet article.

\section{RÉFÉRENCES}

1. Scott RA, Lagou V, Welch RP, et al. Large-scale association analyses identify new loci influencing glycemic traits and provide insight into the underlying biological pathways. Nat Genet 2012 ; 44 : 991-1005.

2. Dupuis J, Langenberg C, Prokopenko I, et al. New genetic loci implicated in fasting glucose homeostasis and their impact on type 2 diabetes risk. Nat Genet $2010 ; 42$ : 105-16.

3. Saxena R, Hivert MF, Langenberg C, et al. Genetic variation in GIPR influences the glucose and insulin responses to an oral glucose challenge. Nat Genet $2010 ; 42$ : 142-8.

4. Yang J, Benyamin B, McEvoy BP, et al. Common SNPs explain a large proportion of the heritability for human height. Nat Genet $2010 ; 42$ : 565-9.

5. Bouatia-Naji N, Bonnefond A, Cavalcanti-Proença $C$, et al. A variant near MTNRIB is associated with increased fasting plasma glucose levels and type 2 diabetes risk. Nat Genet $2009 ; 41: 89-94$.

6. Bouatia-Naji N, Rocheleau G, Van Lommel L, et al. A polymorphism within the G6PC2 gene is associated with fasting plasma glucose levels. Science 2008 ; $320: 1085-8$.

7. Raychaudhuri S, Plenge RM, Rossin हJ, et al. Identifying relationships among genomic disease regions : predicting genes at pathogenic SNP associations and rare deletions. PLoS Genet 2009 ; 5 : p. el000534.

8. Segrè AV, DIAGRAM Consortium, MAGIC investigators, Groop L, et al. Common inherited variation in mitochondrial genes is not enriched for associations with type 2 diabetes or related glycemic traits. PLOS Genet 2010 ; 6 : e1001058

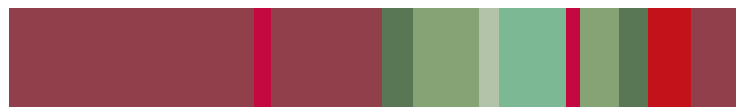

